\title{
Towards 3D 9C Elastic Full Waveform Inversion of shallow seismic wavefields - Case Study Ettlingen Line
}

\author{
T.M. Irnaka ${ }^{1,4}$, R. Brossier ${ }^{1}$, L. Métivier ${ }^{2,1}$, T. Bohlen ${ }^{3}$, Y. Pan $^{3}$ \\ ${ }^{1}$ Univ. Grenoble Alpes, ISTerre, F-38000 Grenoble, France \\ ${ }^{2}$ Univ. Grenoble Alpes, CNRS, LJK, F-38000 Grenoble, France \\ ${ }^{3}$ Geophysical Institute, Karlsruhe Institute of Technology, Germany \\ ${ }^{4}$ Universitas Gadjah Mada, Yogyakarta, Indonesia
}

February 6, 2019

\begin{abstract}
Full Waveform Inversion (FWI) is a high-resolution seismic imaging tool, based on an iterative data fitting procedure. In this research, we focus on a near-surface application using three-component sources and three-component receivers (9C). The target is the Ettlingen Line, a defensive trench line located at Rheinstetten, Germany. In this work, we describe the first application of 3D elastic FWI method to a dense 3D dataset equipped with 9C. In practice, due to the limited number of equipment, the acquisition has been split into six parts (each part has the all source locations but only part of receiver locations). This separation leads to an inconsistent dataset concerning both amplitudes and phases. Therefore, a first step has led to the application of matching filter to homogenize the dataset. Several FWI strategies such as $V_{P}-V_{S}$ parameter binding, gradient Bessel smoothing, and multi-scale approach have been considered during the inversion process to ensure a good convergence. Starting from a homogeneous model, we can achieve significant improvement in data-fit as well as a realistic reconstructed model. The location and dimension of the trench match with the previous experiments based on the inversion of surface wave dispersion curves with an additional increase in resolution.
\end{abstract}




\section{Introduction}

Full Waveform Inversion (FWI) is a high-resolution seismic imaging tool, based on an iterative data fitting procedure. This approach aims at benefiting from all information inside the recorded waveforms. Despite being widely used for crustal exploration, the approach is less popular in shallow seismic. Still, several previous applications at the near-surface scales exist in the literature. For example, Wittkamp et al. (2018) performed 2D FWI on the archaeological target (Ettlingen Line) focusing inversion on the recorded surface wave. Köhn et al. (2018) performed 2D FWI on a shallow engineering target with a significant resolution improvement of the S-wave velocity model $V_{S}$ built from travel time tomography. Smith et al. (2018) also performed 2D and 3D FWI starting from Multi-channel Analysis of Surface Wave (MASW) for $V_{S}$ and travel time tomography for $V_{P}$ to detect the location of the tunnel.

In this research, our target is the Ettlingen Line, a defensive trench line built by German Troops during The War of The Spanish Succession in 1707 (Lang et al., 1907). The trench has been leveled to the ground at the area (Figure 1a). The trench is surrounded by fluviatile sediment deposited from Rhine river. Previously, several studies have been performed for the characterization of this trench. A GPR measurement was performed by Wegscheider (2017) revealing the trench's shape. The size of the trench is approximately six meters in width and five meters in depth. 2D FWI has been performed by Wittkamp et al. (2018), showing low-velocity anomalies at the location of the trench. A more detailed 3D MASW analysis has been carried out by Pan et al. (2018), revealing the 3D shape of the trench.

This study aims at applying a first 3D elastic FWI methodology on a dense 3D dataset equipped with nine components.

\section{Nine components seismic acquisition setup}

Led by GPI KIT, in collaboration with Univ. Grenoble Alpes, GFZ Potsdam, and ETH Zurich; a 3D seismic survey with three-component sources (Figure 1c) and three-component receivers (Figure 1b), 9C Seismic, has been acquired. Dataset from two acquisition grids has been obtained: one coarse and one dense receiver grid. The coarse acquisition has a $2 \mathrm{~m}$ (inline) by $4 \mathrm{~m}$ (crossline) geophone spacing, leading to 128 geophone positions. The dense acquisition has a $1 \times 1 \mathrm{~m}$ geophone spacing, leading to 888 geophones positions. For both receiver grids, the same 36 source locations shown as black dots in Figure 2 have been used. At each location, the Galperin source generates three force sources in orthogonal directions (Häusler et al., 2018).

In practice, such dense receiver grid is challenging for academia with limited available equipment. Therefore, the dense acquisition has been split into six parts (five days). Each part can be identified by a different color-code in Figure $2 b$. For each subset of receivers, we have performed the seismic acquisition for all the source locations.

\section{Data correction with matching filter}

Performing FWI on common-shot-gathers requires a consistent dataset over the shots. For this dataset acquired as six separated subsets (during five days, Figure 2b), 4-D effects associated to uncontrolled
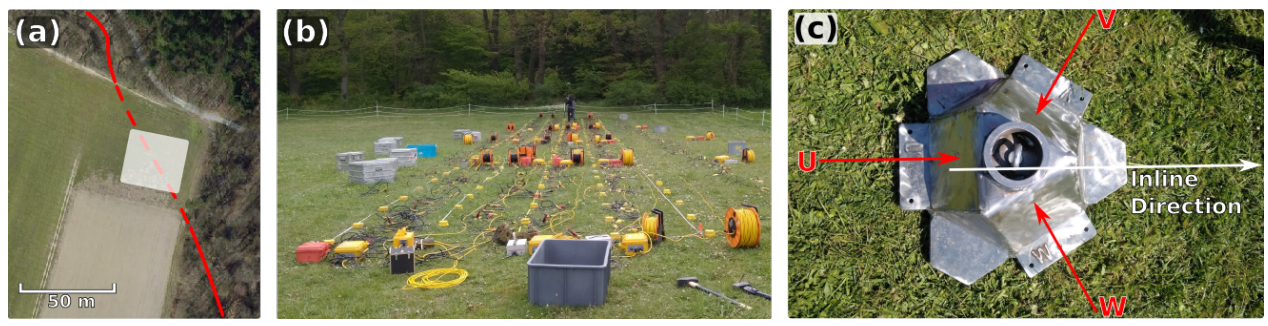

Figure 1: The basemap of Ettlingen Line; solid red line depicts the existing location of the trench line, dashed red line represent the buried site (a). 3D seismic acquisition using 3 component receiver (b) and 3 direction Galperin source (c).

(a)

(b)

Figure 2: Coarse grid acquisition (a) and dense grid acquisition (b). Triangles represent receiver's locations, whereas circles represent source's locations. Different receiver's color shows different acquisition time; $1^{\text {st }}$ day $=$ blue, $2^{\text {nd }}$ day $=$ orange, $3^{\text {rd }}$ day $=$ green, $4^{\text {th }}$ day $=$ red, $5^{\text {th }}$ day $=$ purple and brown. Blue rectangle on the dense grid acquisition represents the seismic line in Figure 3. 


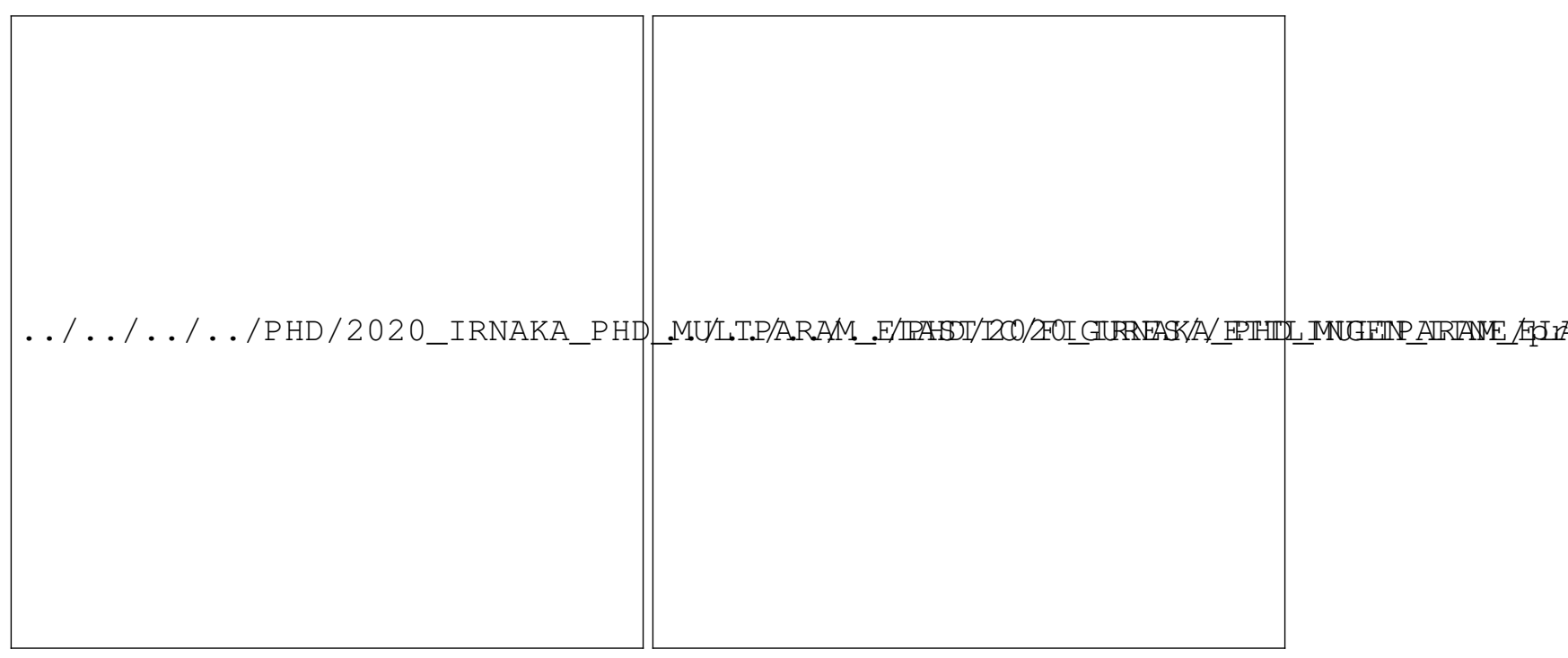

(a) Before data correction.

(b) After data correction.

Figure 3: Seismic data before data correction (a) shows travel time shift between acquisition's subsets, and after data correction (b) where the data is more consistent.

source signature, different source-ground coupling, different ground condition, and a slight change in the equipment setup can contaminate the data. One example of such mismatch between subset is given in Figure 3a, where a small time shift occurs; possibly due to different triggering time. Several other examples such as differences in amplitude and waveform are also observed in the data.

In order to handle such inconsistency, one could have used the six parts independently (requiring six times of 'sources' position). In order to avoid this over-cost, we have chosen to correct the data prior to FWI, thanks to the coarse grid data. On the coarse grid geometry, which matches some dense grid positions, we performed a single acquisition for the whole acquisition area for each source location and direction. Therefore, such reference recordings can be used to compute matching filters to correct the dense grid subsets of data into one consistent dataset.

One matching filter $m_{i, s c, s}(t)$ has been computed for each source location $i$, source component $s c$ (among $U, V, W)$ and subset of data $s$, through a frequency-domain deconvolution

$$
\widehat{m}_{i, s c, s}(\omega)=\frac{\sum_{j, s r} \overline{\hat{d}_{i, s c, s, j, s r}(\omega)} \hat{l}_{i, s c, j, s r}(\omega)}{\sum_{j, s r} \overline{\hat{d}_{i, s c, s, j, s r}(\omega)} \hat{d}_{i, s c, s, j, s r}(\omega)+\varepsilon},
$$

where $\widehat{m}_{i, s c, s}(\omega)$ is the Fourier transform of $m_{i, s c, s}(t), d_{i, s c, s, j, s r}(\omega)$ is the dense grid data associated with the receiver position $j$ and component $s r$, while $l_{i, s c, j, s r}$ is the coarse grid data for the same source/receiver location/component. $\varepsilon$ is the stabilization factor of the matching filter. Once computed, the matching filter is used to correct the dense grid data through convolution, as shown in Figure $3 b$.

\section{FWI setup}

For 3D elastic FWI modeling and inversion, we use the spectral-element SEM46 tool which is presented in Trinh et al. (2019). Spectral element method has been chosen to accurately represent the surfacewave propagation, which is essential for our near-surface application. FWI of shallow-seismic data with limited offset poses several challenges, such as the initial model preparation, relatively high attenuation effect, limited information of the body wave, and strong and dispersive surface wave.

Because of the limited imprint of the body waves and the dominance of the surface wave in the data, we focus our inversion on the surface wave, which is mainly sensitive to $V_{S}$. Therefore, we propose to use a parameter binding strategy to focus on the $V_{S}$ reconstruction, while $V_{P}$ is updated according to a fixed parameter $\alpha\left(V_{P}=\alpha V_{S}\right)$ under a constant Poisson's ratio assumption. Setting this ratio to $v=0.25$ yields the value 1.732 for $\alpha$. The density $\rho$ is kept constant.

We know that the area is surrounded by fluviatile sediment without complex geological structure. Considering this simple geology and rich low-frequency components, we use a relatively simple homogeneous initial velocity model for $V_{S}$. We derive it to be consistent with our parameter binding strategy, 
based on an initial P-wave velocity model built directly from the P-wave travel time at the longest offset of the first inline seismic data on the first source location and direction. This initial homogeneous P-wave velocity is $V_{P}=345 \mathrm{~m} / \mathrm{s}$. Our parameter binding strategy gives us $V_{S}=199 \mathrm{~m} / \mathrm{s}$. A simple Gardner's relation yields $\rho=1336 \mathrm{~kg} / \mathrm{m}^{3}$.

During inversion, source time functions are estimated by matching filter for each source position and component using the Pratt (1999) approach. Gradient smoothing using Bessel filter (Trinh et al., 2017) is applied to reduce the high wavenumber artifact beyond the expected resolution and strong source signature. The inversion is performed with a frequency-continuation approach using six frequency bands, starting from $(3-15) \mathrm{Hz}$ to $(3-65) \mathrm{Hz}$. This multi-scale strategy is crucial in order to avoid cycle skipping on the dispersive surface wave.

\section{First Application of 9C 3D elastic FWI for Ettlingen Line}

Starting from a homogeneous model, we can reconstruct $V_{S}$ model to some details with significant improvement of data fit. Figure 4 shows the data fit for the initial model and the reconstructed model in the

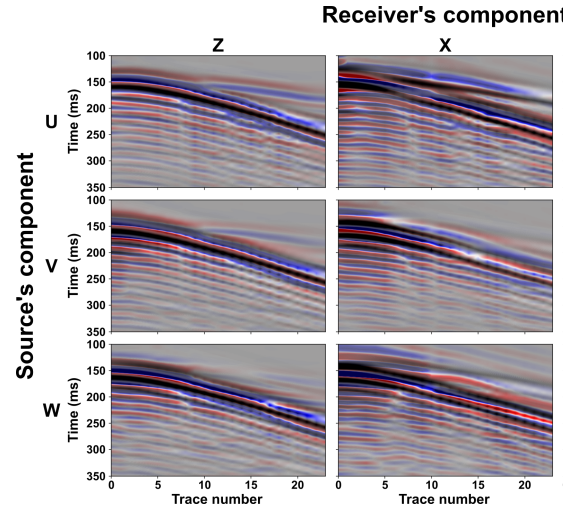

(a) Initial model.
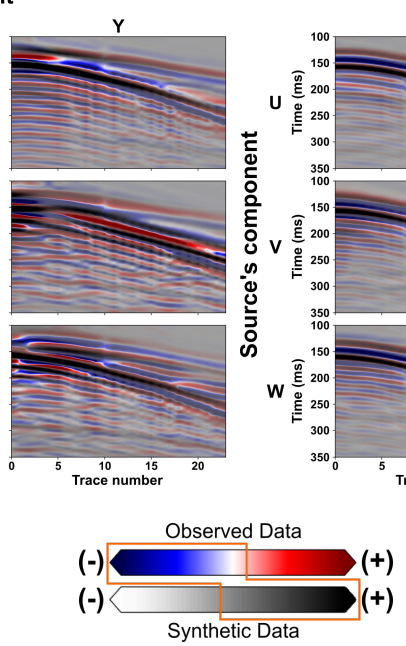

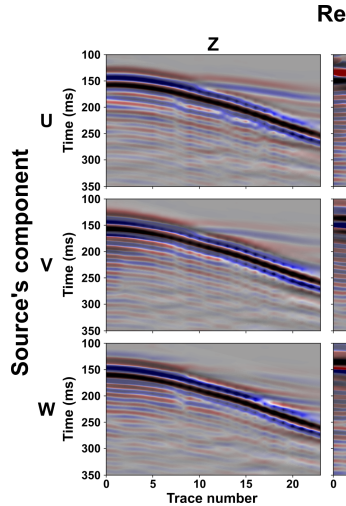

Receiver's component

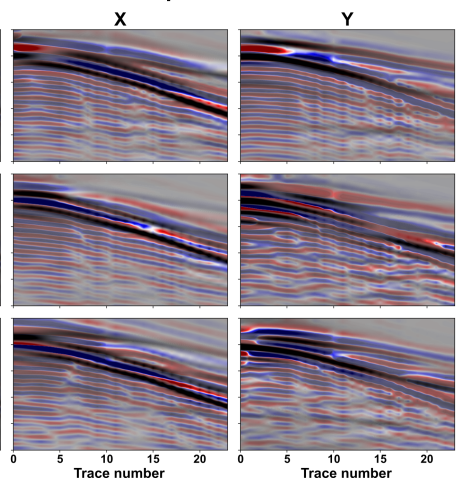

(b) Reconstructed model.

Figure 4: Data misfit on the initial model (a) and the reconstructed model (b). The given color scale at the bottom represent both the observed and synthetic data. A good misfit is denoted by combination of dark blue and black.
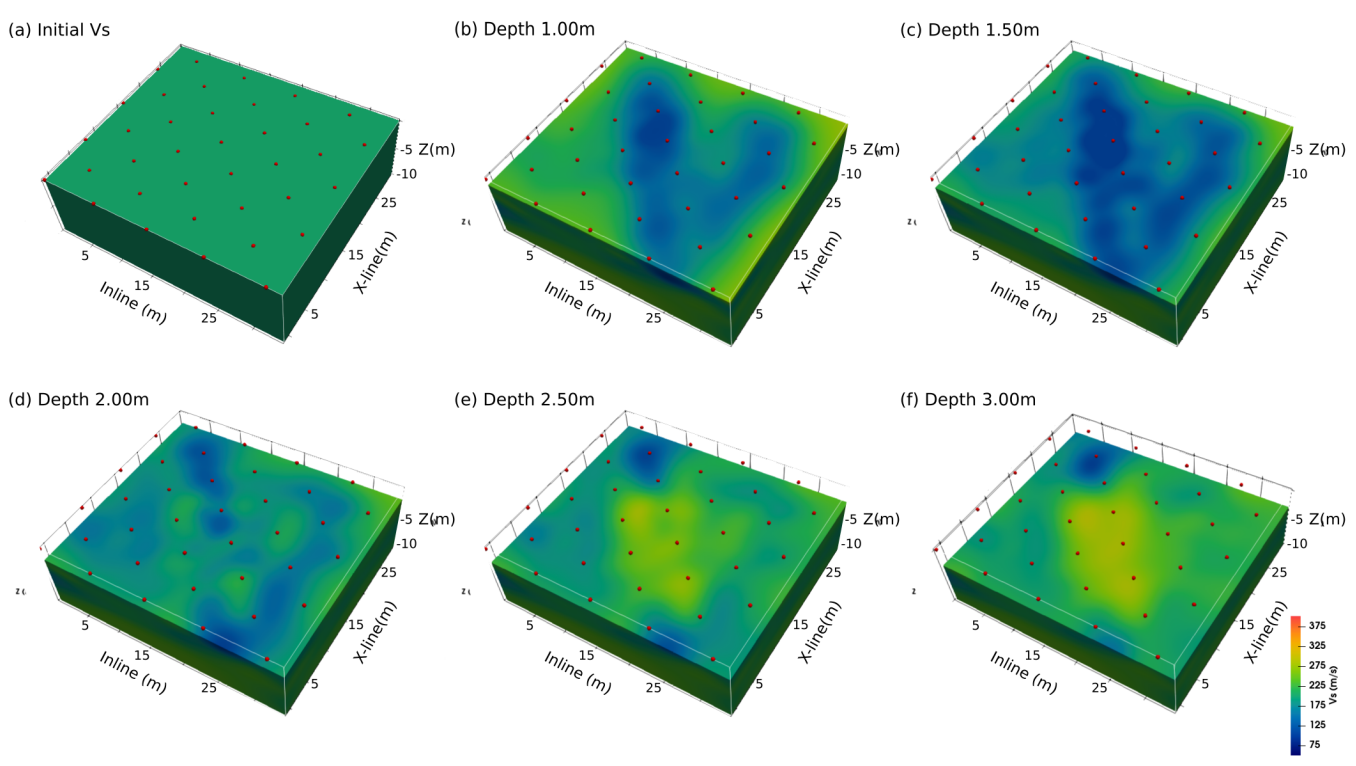

Figure 5: Initial (a) and the reconstructed $V_{S}$ model from various depth (b-f). Red dots on each figure represent source's locations. 
(3-65)Hz frequency band. The plot is the superposition between the observed data (blue-red color scale) and synthetic data (white-black color scale). The data fit can be considered as good if the plot mainly contains blue and black color. On the data from the initial model, significant travel time mismatches are observed on all components. After inversion, a fairly good fit is observed on all arrivals. Although we expect a high attenuating medium, it seems we can still predict the observed data using the elastic approach in this frequency band.

Figure 5 shows several depth slices of the reconstructed $V_{S}$ model on the highest frequency band (Figure $5 b-f)$. The Ettlingen Line is generally characterized by a low-velocity zone with direction northwestsoutheast. This result is consistent with the previous studies in this area. In general, the Ettlingen Line's width at the surface is around 3 to $5 \mathrm{~m}$, whereas its depth is around 2 to $2.5 \mathrm{~m}$. Heterogeneities inside the trench are mostly observed at a depth of $1.5 \mathrm{~m}$ (Figure 5d) which might represent the variation of soil's characteristics (such as compaction level).

Beside the Ettlingen Line, we also observe another low-velocity zone with a north-south orientation. The width of this trench-like structure is around $5 \mathrm{~m}$ at the surface, and the depth is around $2 \mathrm{~m}$. It is generally shallower than the Ettlingen Line. We currently do not know the historical significance of this second trench-like structure.

\section{Conclusions and Outlooks}

This study presents a 3D elastic FWI using 9C on a dense dataset for a near-surface archaeological target. This FWI has been performed by carefully preprocessing the data through matching filters, $V_{P^{-}}$ $V_{S}$ parameter binding, Bessel gradient smoothing, and multi-scale strategy. It enables us to predict the observed data with significant improvement. A high-resolution $V_{S}$ structure has been reconstructed from a homogeneous background, giving us the 3D details of the Ettlingen Line's geometry as well as another trench-like structure with a north-south orientation. Further studies regarding the detailed effect of the initial model design, the attenuation, and multi-parameter inversions will be conducted in the future.

\section{Acknowledgements}

The authors thank the collaboration between GPI KIT, UGA, GFZ Potsdam, ETH Zurich which enable us to perform the acquisition, processing, and FWI. This study was partially funded by the SEISCOPE consortium (http://seiscope2.osug.fr), sponsored by AKERBP, CGG, CHEVRON, EQUINOR, EXXON-MOBIL, JGI, PETROBRAS, SCHLUMBERGER, SHELL, SINOPEC, and TOTAL; also partial financial support by the Deutsche Forschungsgemeinschaft (DFG) through CRC 1173. This study was granted access to the HPC resources of CIMENT infrastructure (https://ciment.ujf-grenoble.fr) and CINES/ IDRIS/TGCC under the allocation 046091 made by GENCI.

\section{References}

Häusler, M., Schmelzbach, C. and Sollberger, D. [2018] The Galperin source: A novel efficient multicomponent seismic source. Geophysics, 83(6), P19-P27.

Köhn, D., Wilken, D., De Nil, D., Wunderlich, T., Rabbel, W. and Werther, L. [2018] 2D full waveform inversion applied to a strongly-dispersive Love wave field dataset. In: 80th EAGE Conference and Exhibition 2018.

Lang, K. et al. [1907] Die Ettlinger Linien und ihre Geschichte. Selbstverlag der Stadt Ettlingen.

Pan, Y., Schaneng, S., Steinweg, T. and Bohlen, T. [2018] Estimating S-wave velocities from 3D 9-component shallow seismic data using local Rayleigh-wave dispersion curves - A field study. Journal of Applied Geophysics, 159, 532-539.

Pratt, R.G. [1999] Seismic waveform inversion in the frequency domain, part I : theory and verification in a physical scale model. Geophysics, 64, 888-901.

Smith, J.A., Borisov, D., Cudney, H., Miller, R.D., Modrak, R., Moran, M., Peterie, S.L., Sloan, S.D., Tromp, J. and Wang, Y. [2018] Tunnel Detection At Yuma Proving Ground, Arizona, USA. Part 2: 3D Full-Waveform Inversion Experiments. Geophysics, 84(1), 1-98.

Trinh, P.T., Brossier, R., Métivier, L., Tavard, L. and Virieux, J. [2019] Efficient 3D time-domain elastic and viscoelastic Full Waveform Inversion using a spectral-element method on flexible Cartesian-based mesh. Geophysics, 84(1), R75-R97.

Trinh, P.T., Brossier, R., Métivier, L., Virieux, J. and Wellington, P. [2017] Bessel smoothing filter for spectral element mesh. Geophysical Journal International, 209(3), 1489-1512.

Wegscheider, S. [2017] Abbildung der Ettlinger Linie auf dem Segelflugplatz Rheinstetten mittels Georadar. Master's thesis, Karlsruhe Institute of Technology.

Wittkamp, F., Athanasopoulos, N. and Bohlen, T. [2018] Individual and joint 2-D elastic full-waveform inversion of Rayleigh and Love waves. Geophysical Journal International, 216(1), 350-364. 